\title{
GLAD!
}

Revue sur le langage, le genre, les sexualités

Archives, genre, sexualités, discours

\section{Les archives, un outil dans la construction d'un rapport de force féministe : l'exemple d'un groupe féministe et syndical}

Records, a Tool in the Construction of a Feminist Power Struggle: The Example of a Feminist Union Group

\section{Maëva Ballon et Flaz Torres}

\section{OpenEdition \\ Journals}

Édition électronique

URL : https://journals.openedition.org/glad/3402

ISSN : 2551-0819

Éditeur

Association GSL

Référence électronique

Maëva Ballon et Flaz Torres, «Les archives, un outil dans la construction d'un rapport de force

féministe : l'exemple d'un groupe féministe et syndical », GLAD! [En ligne], 11 | 2021, mis en ligne le 20 décembre 2021, consulté le 29 janvier 2022. URL : http://journals.openedition.org/glad/3402

Ce document a été généré automatiquement le 29 janvier 2022.

\section{cc) (오 $\ominus$}

La revue GLAD! est mise à disposition selon les termes de la Licence Creative Commons Attribution Pas d'Utilisation Commerciale - Pas de Modification 4.0 International. 


\title{
Les archives, un outil dans la construction d'un rapport de force féministe : l'exemple d'un groupe féministe et syndical
}

\author{
Records, a Tool in the Construction of a Feminist Power Struggle: The Example of \\ a Feminist Union Group
}

Maëva Ballon et Flaz Torres

\section{NOTE DE L'AUTEUR}

Nous sommes des autrices. A chaque fois que le mot « auteur » sera utilisé pour nous désigner, nous demandons aux lectrices et lecteurs de lire " autrices».

En mai 2018, nous avons été à l'initiative de la création d'un groupe féministe au sein de notre syndicat. Suspendu par anti-féminisme par notre direction syndicale en juin 2020, notre groupe a néanmoins été actif jusqu'en mars 2021.

\section{Introduction}

1 Militantes féministes et syndicales, notre groupe a été créé par décision de l'assemblée générale de notre syndicat en 2018, par un vote d'assemblée générale des syndiqué·es, à l'unanimité moins un. L'enjeu : aborder le sujet de l'égalité au travail entre les femmes et les hommes et la lutte contre les discriminations à l'égard des personnes LGBTQI d'un point de vue syndical. Dès sa création, le groupe s'est donné pour objectif de "définir des revendications concrètes autour de cette thématique transversale afin d'ouvrir des négociations et faire de l'égalité une réalité ». Ouvert à toutes et tous, celui-ci est composé de camarades actifives. Certaines sont élues du personnel, d'autres sont impliquées dans les activités et les sections de travail permanentes de 
notre syndicat. La démarche de transmission des réflexions et pratiques communes à notre groupe féministe s'inscrit dans une perspective de partage, de sororité et de combat pour l'égalité.

2 La production, l'utilisation et la conservation de nos archives se situent dans différentes échelles de temps et d'espaces politiques. Par l'exemple d'objets militants, témoins du fonctionnement et de l'activité menée par notre groupe pendant près de trois ans, nous chercherons à démontrer l'importance des archives dans la construction d'un rapport de force féministe.

3 Être féministe et militer pour la cause féministe, c'est d'abord faire corps ensemble, par la présence physique ou virtuelle. Aussi, dans notre groupe, les modalités de production des archives ${ }^{1}$, entendues comme des marqueurs des intentions militantes, sont aussi importantes que leur finalité. Interroger en amont les conditions de production des archives consiste à envisager d'un point de vue féministe les contraintes genrées de l'activité militante ainsi que les rapports d'oppression subis par les femmes au sein de notre organisation syndicale. Les choix de support de nos productions, mais aussi d'élaboration et de partage de nos comptes rendus de réunion en temps réel permettent de mieux appréhender les enjeux en cours : laisser une trace sur l'objet lui-même et sur la manière dont il nous semble utile de le partager, limiter les contraintes de temps des militantes pour créer une archive commune sans investissement postérieur supplémentaire ou encore réduire les barrières liées au côté initiatique de l'intégration au groupe. Au final, le partage, la construction collective, la confiance, la sororité et l'horizontalité sont autant de composantes intrinsèques des pratiques militantes féministes, dont l'élaboration des archives constitue le témoin privilégié.

4 Dans notre fonctionnement, il n'existe pas de trace produite pour ne pas être utile. Nos archives favorisent la légitimité de nos revendications féministes internes à notre organisation, mais aussi face à notre employeur. Démarche collaborative ou preuve de la prise d'une décision, les archives sont au cœur de la construction du rapport de force en tant qu'outils de médiation directe et authentique de nos engagements. Ainsi, l'ensemble des camarades de la commission exécutive de notre syndicat dispose d'un compte rendu de nos réunions de groupe décrivant les objectifs, les modalités d'actions retenues, les actions, les dates et le rappel des validations précédentes.

5 En archivistique, le contexte de production et les modes d'utilisation de l'objet sont aussi importantes que l'objet lui-même. De même, sur le plan militant et féministe, nous ne concevons pas le partage d'un tract, d'une affiche syndicale ou de toute autre production militante comme une finalité en soi, mais bien dans une totalité où les pratiques de conception et d'utilisation sont intrinsèquement liées à l'archive ellemême, au sens où le partage de nos motivations, nos choix de forme et nos processus d'élaboration participent de la caractérisation de nos objets militants comme des archives.

6 Nous appuyer sur le numérique tant comme technologie que comme base de supports pivots de nos productions, nous conduit nécessairement à questionner, d'une part, nos usages au regard de la répartition inégale des capitaux entre nous et d'autre part la fragilité des socles techniques sur lesquels nous élaborons nos dispositifs et qui conditionnent la pérennité des traces de l'action féministe. Si les enjeux de sauvegarde et de transmission sont consubstantiels de l'archivistique actuelle, ils s'imposent aussi dans nos temporalités militantes. Dès la création de notre groupe féministe, la 
conservation à long terme de nos écrits papiers et numériques a été réfléchie, comme le reflète la réalisation d'un wiki, ou site web collaboratif, dès nos premiers échanges militants. Dans ce contexte, les pratiques féministes de constitution de nos espaces de mobilisation en espaces de production et de partage de savoirs sont une ressource essentielle.

\section{Les comptes rendus de réunion de groupe}

Wiki de notre groupe féministe et syndical, copie d'écran de la rubrique «Anciennes réunions », mai 2021

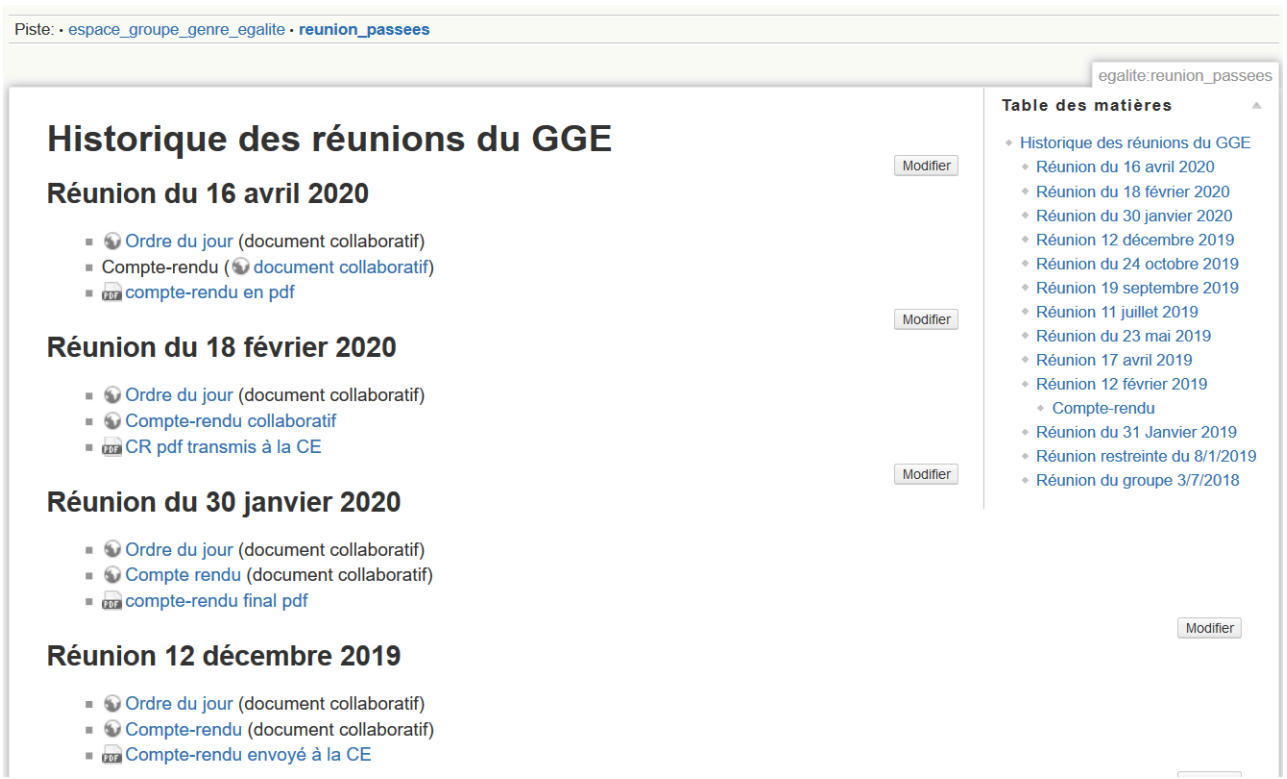

7 Objets d'archives en mouvement permanent, les comptes rendus de réunion de notre groupe représentent le socle textuel de mise en commun de notre activité militante et féministe. Les réflexions et les pratiques, de la construction de nos ordres du jour jusqu'au partage et à la communication de nos comptes rendus, illustrent les mécanismes de sororité et d'entraide que nous avons tenté de mettre en place pour neutraliser l'instantanéité et le fonctionnement pyramidal propres à la masculinité hégémonique ${ }^{2}$, théorisée par la sociologue Raewyn Connell dès $1995^{3}$.

Impossible d'évoquer le compte rendu sans discuter en amont de ce qui l'a précédé. Plusieurs jours avant chaque réunion, un ordre du jour est proposé par un·e camarade. Élaboré sur la base des priorités militantes décidées collectivement, il fait également apparaître les points que nous n'avons pas eu le temps d'aborder lors des précédentes réunions. En réinscrivant à chaque instant notre calendrier militant dans l'histoire et la dynamique de nos échanges, c'est-à-dire dans une continuité où nos actions passées et leur processus d'élaboration nous permettent de comprendre et d'élaborer nos actions présentes, nous laissons la place à nos réflexions antérieures. Cette règle permet de prendre de la distance sur nos choix d'activités, dans un contexte social et syndical marqué par la sur-sollicitation et l'excès d'information. Ce rappel de nos échanges passés s'inscrit aussi dans la lutte contre les rapports de domination et de hiérarchisation interne des sujets militants ${ }^{4}$ et l'accaparement de nos actions par des militantes habituées du syndicalisme et de ses formalismes tacites. Aucune idée ou 
proposition non discutée faute de temps, n'est écrasée ou oubliée. Conscientes des contraintes temporelles et en investissement des femmes de notre groupe, il s'agit d'un rempart face à nos propres biais. Pour créer ensemble sans distinction ou prérequis, et pallier les défauts d'un envoi par mail à amender individuellement, toutes nos réunions débutent par une discussion autour de la validation de l'ordre du jour.

Guidé·es par la conviction que nous sommes plus fort·es quand nous sommes ensemble, nous élaborons en direct nos comptes rendus de réunion. La restitution immédiate et synthétique de nos propos s'inscrit ainsi dans une dimension collective avec une vocation de diffusion instantanée. L'utilisation des pads pour l'ensemble de notre production d'archives textuelles est facilitatrice. Éditeurs numériques de texte collaboratif en ligne, ils permettent d'écrire à plusieurs en direct. Les mots sont visibles par toutes sur un écran partagé ou un ordinateur individuel. Ils peuvent être modifiés à tout moment, sans investissement temporel supplémentaire. Leur accessibilité reste néanmoins soumise à certaines conditions d'appropriation. Les pads ne suppriment pas les barrières du passage à l'écrit, ni celles de l'assimilation d'une pratique qui bouleverse les habitudes. Pour y remédier, nous essayons de présenter à chaque réunion du groupe une fonctionnalité particulière d'un outil numérique. Il s'agit d'une étape de construction collective basée sur l'apprentissage.

10 Notre groupe fait partie d'une organisation hiérarchique. Si la prise de décisions dans notre syndicat s'effectue le plus souvent lors de réunions physiques et formelles des débats d'orientation des camarades, nos comptes rendus laissent une trace et renforcent la légitimité de nos arguments et de nos propositions d'actions. En tant que groupe féministe, nous devons davantage justifier nos actions et argumenter pour être entendues. Nos comptes rendus participent du rapport de force interne au sein de notre organisation. Ils nous donnent de la force dialectique, inscrivent nos actions et nos propositions dans la durée. Il nous arrive d'y faire appel pour lancer un débat ou pour motiver une activité militante. Par exemple, alors que la direction de notre syndicat remettait en cause le bien-fondé de réaliser une enquête sur les violences sexistes, sexuelles et lgbtphobes auprès de nos collègues, nous nous sommes appuyé es sur le compte rendu de la précédente réunion de commission exécutive qui actait le lancement de l'enquête pour déjouer les manœuvres visant à enliser l'élaboration collective sur le sujet, à contester notre légitimité à nous appuyer sur des décisions prises ou simplement à nous épuiser. Si nos archives de réunions ne suffisent pas en soi à constituer un instrument argumentatif et de légitimation, elles participent, par leur nombre, leur régularité et leur qualité, de l'occupation de l'espace syndical par les questions féministes.

11 En outre, finalisés, nos comptes rendus de réunion sont des outils indispensables pour préparer nos interventions officielles. Archives de partage et d'anticipation, ils ne sont pas des consignes de lecture verticale mais bien des objets aux services des camarades, dont iels peuvent se saisir à tout moment. Dans un contexte militant marqué par une sur-charge de sollicitation et d'implication individuelle, ils représentent notre mémoire pour argumenter face à notre employeur. Dans le cadre de réunions de présentation du plan de labellisation AFNOR (Agence française de normalisation) en matière d'égalité professionnelle organisées par notre employeur, par exemple, nous nous sommes appuyées sur nos comptes rendus pour construire notre réflexion et préciser nos arguments à l'aune des notes prises en cours de réunions employeur où aucun document n'était fourni en amont pour préparer et travailler les sujets. 
12 Enfin, leur préservation est corrélée à leur accessibilité et leur appropriation par toutes. Dans la mesure du possible, nous conservons les pads modifiables des ordres du jour initiaux. Cela répond à deux objectifs pratiques: ne pas perdre de temps dans l'élaboration d'un nouvel ordre du jour ; partager et rendre accessible le fruit de notre travail, dans un souci d'entraide et d'horizontalité de nos pratiques. La mise à disposition des comptes rendus de réunion dans un unique endroit de stockage en ligne permet, même s'il est peu consulté, de travailler en confiance et de lutter contre les difficultés à s'initier seule à la culture d'organisation du syndicat ${ }^{5}$. Les nouvelles et nouveaux camarades savent qu'iels peuvent retrouver l'information sur nos choix d'organisation ou de campagnes militantes. Nos comptes rendus constituent à ce titre des objets de mémoire, au sens " des choses qui ont été dites " ${ }^{6}$ à travers notre activité de groupe. Ils sont notre matrimoine dont nous nous servons pour produire notre bilan d'activités.

\section{Un tract}

13 La production collaborative de traces est un levier pour la réalisation d'outils militants dans une démarche féministe. Ainsi, la création d'un tract impose d'apporter des réponses concrètes à de nombreuses questions politiques imbriquant le contenu du tract, les personnes auxquelles il est destiné, le type d'action auquel il contribue et les personnes qui vont le tracter. On l'oublie trop souvent, un tract féministe est fait pour être tracté, c'est-à-dire passé en face à face, de la main à la main, ouvrant une opportunité d'interaction entre un·e militant·e et une personne à laquelle elle s'adresse. Que l'on se pose explicitement les questions ou non, on est contraint·es d'appréhender cette potentialité d'engagement dans l'échange, une forme affirmative d'occupation et de prise de parole dans l'espace public et, sur le lieu de travail (à la différence des lieux de tractage " anonymes »), les effets différés positifs (interactions avec les collègues) ou négatifs (répression) de cette exposition. L'action militante sur le lieu de travail engage de manière différente la crédibilité des militantes du fait d'une continuité dans l'itération des interactions avec des personnes connues et manifeste un niveau d'engagement qui n'échappe pas à la hiérarchie. Or, il est impossible de prendre en compte toutes ces dimensions si elles ne sont pas anticipées dès l'élaboration de l'action et dans la rédaction. Une attention particulière est portée aux processus délibératifs et décisionnels (participation, énonciation-retranscription) pour qu'ils ne soient pas excluants en termes de disponibilité générale et de synchronicité.

L'objet tract est ici appréhendé sous l'angle d'un ensemble de pratiques enchâssées dans des rapports sociaux de sexes ordinairement marqués non seulement par l'appropriation collective du travail militant des femmes par les hommes mais aussi par l'appropriation individuelle par les dirigeants du travail de certaines femmes ${ }^{7}$. Bien qu'elle soit toujours à l'œuvre, elle est particulièrement établie et normalisée dans des structures syndicales régies par un fonctionnement bureaucratique, pyramidal, patriarcal et clanique. Dans ce type de structure qui est le nôtre, l'image prévalente, explicitement mobilisée par les dirigeant·es est celle de la famille ${ }^{8}$ où l'appropriation par le patriarche s'énonce comme "valorisante" quand elle prend la forme d'une faveur accordée à certaines militantes, les plaçant dans une position de favorites tout en les dépossédant de leur travail (réécriture le plus souvent destructive) et de la majeure partie, voire de la totalité, de la reconnaissance de leur travail. 
production de traces d'élaboration qui peuvent être très antérieures, dans une dimension projective ${ }^{9}$ de l'action que permet une «campagne» ou circonstancielle ${ }^{10}$ pour ce qui relève de l'intention poursuivie, de l'utilisation que l'on compte en faire, de son articulation à d'autres productions. Par exemple, le 8 mars 2020, notre groupe a organisé une semaine de l'égalité scandée par une performance, une projection débat et un appel à la manifestation parisienne. Elle a donné lieu à la réalisation d'une affiche, des paroles d'une chanson, de deux tracts recto-verso et d'un mail de rendez-vous au sein de la manifestation. Ces productions venaient en appui d'actions de terrain dans lesquelles les membres du groupe étaient directement engagées et motrices ${ }^{11}$.

Les tracts que nous produisons s'inscrivent dans une action collective. On ne fait pas « un tract pour le 8 mars ». Si on décide d'en rédiger un, ce sera parce que cet outil nous semble utile pour les actions que nous mènerons à l'occasion de cette échéance de mobilisation et afin de l'inscrire dans notre agenda local, féministe et syndical. Nous arrimons nos revendications locales à celles portées par le mouvement féministe international.

Ce temps de mobilisation s'est d'emblée construit à partir de sources d'archives féministes : le film-documentaire Je ne suis pas féministe mais... ${ }^{12}$, le kit multimédia de la chorégraphie réalisée par le groupe Les Rosies, "À cause de Macron», les différents appels syndicaux et associatifs. Il a donné lieu à la production de nombreux écrits à vocation interne retraçant les choix d'élaboration et les débats : comptes rendus de réunions, choix de maquettes, options graphiques, plans, textes, repérage rythmique qui constituent, par leur nombre et leur diversité autant d'objets écrits de légitimation de notre travail. Ils donnent à voir la multiplicité et la diversité des actions menées tout en permettant de se saisir des choix effectués et des débats qui y ont présidé.

\section{Une chanson}




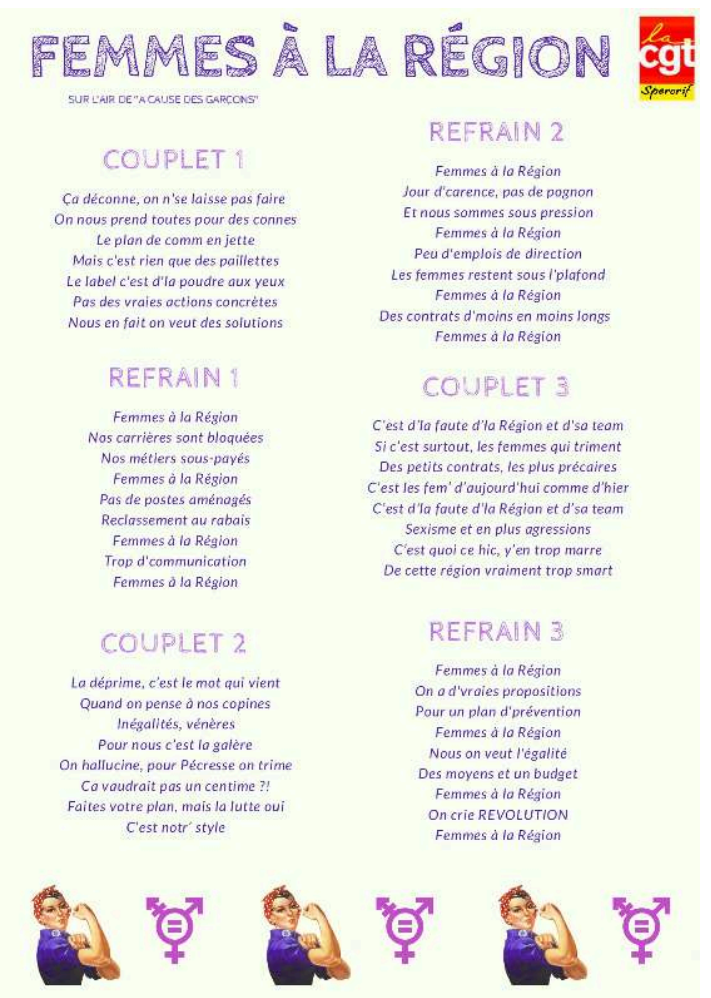

La création et l'interprétation d'une chanson féministe sur notre lieu de travail représentent un exemple singulier d'objet d'archives militant comme point d'appui dans la construction d'un rapport de force féministe.

En choisissant un air, "À cause de Macron", issu du mouvement social féministe contre la réforme des retraites de 2019 et 2020, nous donnions de la force à nos propos. En l'interprétant à l'occasion du 8 mars 2020, nous nous inscrivions dans un contexte historique et international. En exploitant le kit des Rosies, nous revendiquions l'aspect matrimonial de notre démarche.

Tout, de l'idée initiale, des nouvelles aptitudes qu'elle implique, de son processus d'élaboration, puis d'interprétation, constitue des marqueurs de nos pratiques féministes. Pour dénoncer l'inaction de notre employeur en matière d'égalité entre les femmes et les hommes, il fallait gagner en visibilité tout en associant nos collègues. Pour être entendues et faire bouger les lignes dans notre contexte syndical, nous cherchions à sortir de nos habitudes militantes et à concevoir un outil percutant.

Dans nos réalités de travail et de militance, la chanson casse les codes des formalismes et des pratiques syndicales traditionnelles. Depuis plusieurs années, un processus de neutralisation des espaces de visibilité des organisations syndicales est en marche sur notre lieu de travail. Les panneaux d'affichage sont moins nombreux, les locaux syndicaux de plus en plus petits et les grandes salles de réunion pour échanger et débattre avec les collègues de moins en moins accessibles. Les temps de rencontre sont réduits du fait de l'augmentation de la flexibilité des horaires de travail et de la généralisation du télétravail pour une partie de nos collègues. À cet encadrement limitatif du droit d'information syndicale s'ajoutent les avertissements menaçants de notre employeur à l'occasion de mouvements locaux de grève et de mobilisation. La direction de notre syndicat a, à plusieurs reprises, été « invitée » à stopper toute action 
«bruyante ». L'usage du mégaphone dans les espaces publics communs est décrié par notre employeur. En choisissant la chanson comme objet militant, nous souhaitions non seulement lutter contre la répression de l'expression syndicale, mais aussi occuper les espaces de travail et militants. Notre objectif était de réaliser une performance en travaillant sur l'engagement physique comme briseur de glace auprès des collègues. Nous affirmions ainsi que le féminisme a sa place partout.

Notre chanson militante impliquait des aptitudes que nous avions peu l'habitude d'utiliser tels que le chant, la danse ou la recherche de déguisements. En s'appuyant sur ces registres de compétences moins ordinaires et plus populaires, propres à cet objet d'archives, nous permettions l'expression de toutes les camarades, sans hiérarchie des formes de mobilisation. Nous nous sommes réunies à plusieurs reprises sur la pause déjeuner pour écrire les paroles collectivement et répéter la chorégraphie. Sa réalisation fut un réel moment de plaisir et de confiance mutuelle. Les paroles fusaient en même temps que les camarades se sentaient libres de partager leurs idées et leurs expériences.

\section{Midis Féministes}

Les midis féministes sont conçus comme un rendez-vous convivial où l'on partage le moment du repas en même temps que des idées, des expériences... L'intention affichée dans notre communication écrite est «de prendre du plaisir à se retrouver entre collègues pour échanger sur des questions qui nous touchent et nous intéressent ».

Par la simple mobilisation de la notion de plaisir, cette forme d'action tranche avec les ressorts usuellement sollicités «en externe " par notre syndicat, y compris par notre groupe. Cette singularité va de pair avec une spécificité d'inscription dans notre plan d'action militant en regard des autres actions, programmées ou non, que nous nous attachons à articuler autant que nous le pouvons. À l'issue d'une délibération collective, nous avons décidé que les midis féministes n'avaient pas de continuité thématique ni de contrainte d'inscription dans notre activité militante ou l'actualité féministe au sens large. Chaque midi met en discussion un sujet fixé à l'avance (la santé des femmes, les inégalités de patrimoines, la répartition des tâches ménagères...).

La latitude réelle dont dispose les agent·es lors de la pause méridienne nous a conduites à fixer à une heure la durée de chaque midi féministe. Ce choix limitatif fut dicté par un impératif de non-discrimination vis-à-vis des agent-es contraintes par la surveillance hiérarchique ou par la nécessité de limiter la durée de présence sur le lieu de travail en raison de la double-journée ou des temps de transport. À la rencontre d'un objectif politique (échanger à bâtons rompus en partant d'une contribution réflexive structurée) et d'une exigence pratique (déjeuner et discuter), les midis sont construits sur une structure classique : introduction puis discussion. L'objectif étant d'échanger, la partie introductive ne peut dépasser 20 minutes.

Par construction, les midis féministes tels que nous les souhaitons ne peuvent exister qu'en s'appuyant sur des archives féministes, textuelles, sonores ou audiovisuelles ${ }^{13}$. Explorer les archives féministes, se familiariser avec les sources et s'en nourrir est donc une activité de fond essentielle à l'existence des midis. Comme dans la plupart de nos actions, ce recours à des sources s'accompagne d'une appropriation selon plusieurs modalités. 
27 L'échange délibératif entre les membres du groupe permet de mettre en partage les motivations qui nous poussent à proposer une thématique et un support pour l'introduire ainsi que les recherches éventuelles que nous avons faites pour explorer les angles permettant d'introduire la thématique. La levée de toute contrainte " militante » (au sens d'un impératif d'efficacité caractérisé) facilite la mise sur le même plan, dans l'échange d'idées jusqu'à la décision, de connaissances théoriques, de connaissances tirées d'expériences militantes passées, de connaissances issues d'expériences individuelles ou collectives à travers des sociabilités affinitaires ou familiales.

Quelle que soit la nature de la source originale (texte, vidéo, audio), il nous faut intervenir sur les documents afin d'en extraire un apport qualitatif, limité à $20 \mathrm{~min}$ (coupes, remontage...). Si le travail effectif de sélection est le plus souvent individuel, les résultats intermédiaires sont "capturés " et mis en débat, permettant une appropriation par le groupe de la séquence d'introduction. Il s'agit tout autant d'aiguiser l'angle d'approche que de décider quels propos anti-féministes nous mettons en débat, dans un temps aussi limité. En pratique, nous avons été conduites à produire des ressources dérivées, adaptées à l'espace féministe que nous souhaitions créer.

La lecture à plusieurs voix d'un texte écrit a d'emblée été envisagée lors du lancement des midis. Lorsque nous avons souhaité l'expérimenter, nous avons été confrontées à deux types d'obstacles : difficultés ou réticences à prendre la parole dans le cadre d'une lecture publique, disponibilité de certaines lectrices le jour J. À l'opposé du repli sur les "plus maîtresses de leur emploi du temps » et les "plus à l'aise " (discriminations socioculturelles) nous avons fait le choix de la réalisation d'un enregistrement à plusieurs voix ${ }^{14}$. Après découpage et répartition, chacune était en mesure de répéter et de s'enregistrer autant que souhaité. La question de la continuité de tonalité a été tranchée en amont, laissant chaque lectrice libre de restituer oralement sa propre interprétation du texte, dans une fidélité absolue à la lettre. Les fragments sonores ont été mis en partage afin que chacune dispose d'un retour extérieur, dans l'espace d'échange bienveillant du groupe. Après quelques itérations, cette mise en partage nous a permis de valider collectivement l'ensemble des fragments avant le montage final. L'exercice s'est mécaniquement traduit par une très forte appropriation du texte et par la restitution d'une diversité d'interprétations politiques et artistiques.

30 Le résultat ${ }^{15}$ a non seulement pu être diffusé le jour $\mathrm{J}$ mais nous l'avons rendu disponible en téléchargement public et documenté, avec l'accord de l'autrice du texte original.

\section{Une enquête sur les violences sexistes, sexuelles et Igbtphobes au travail}

31 La réalisation d'une enquête sur les violences sexistes, sexuelles et lgbtphobes permet de produire de la connaissance et de la partager. Dans notre contexte syndical, ce savoir représente un point d'appui féministe pour porter des revendications avec nos collègues et féminister notre syndicat en révélant les réalités niées des violences subies par les femmes. La construction du document d'analyse des résultats ${ }^{16}$ est emblématique de notre démarche, à l'opposée d'une apparente expertise scientifique. Il ne s'agit pas d'une succession de données chiffrées, mais bien d'une mise en lumière 
d'enseignements et de revendications. Leur transmission sert avant tout d'outil de sensibilisation, d'interpellation et d'appropriation.

L'élaboration du questionnaire d'enquête s'inscrit dans une histoire de partage et de sororité. Nous nous sommes saisies de plusieurs questionnaires réalisés par des camarades d'autres structures. Avant d'entrer dans la phase d'élaboration proprement dite, nous avons étudié des questionnaires disponibles sur des sites syndicaux. Nous nous sommes appuyées sur le questionnaire produit par les camarades du collectif féministe national de notre organisation ${ }^{17}$. Ce choix répondait notamment à la volonté de mener notre enquête de manière conjointe avec un autre syndicat de la même confédération ${ }^{18}$ intervenant sur le même lieu de travail que nous ${ }^{19}$. Nous avons cependant dû adapter ce questionnaire aux spécificités de notre structure de travail de manière à pouvoir étudier d'éventuels effets structuraux. De plus, nous avons ajouté la dimension des violences à l'égard des personnes LGBT car notre groupe féministe intervenait depuis sa création sur ces deux dimensions de l'oppression patriarcale. Enfin, nous avons affiné les questions de manière à faciliter les réponses des collègues et enrichir l'analyse. Nous inscrivions ainsi notre action dans une histoire du féminisme syndical, refusant le simple « copier-coller » utilitariste.

Cette démarche autour d'un objet a permis de nombreux rapprochements, une entraide dans la diffusion et la collecte et a servi d'échappatoire à l'entre-soi militant. En multipliant les rencontres, nous avons non seulement amélioré notre questionnaire, mais aussi pris de la distance avec notre quotidien militant. Une vraie respiration.

Les modalités de diffusion de notre enquête ont été pensées dans un souci de nondiscrimination. Pour permettre l'accessibilité et la prise en main par toutes et tous, nous avons utilisé un outil d'enquête centralisateur en ligne, un support de questionnaire numérique mais aussi un support papier. Ce dernier, par sa matérialité, a également participé de l'occupation de l'espace syndical par les questions féministes. En effet, nous avons utilisé un des droits annuels syndicaux d'impression et de diffusion par notre employeur d'un 4 pages à l'ensemble du personnel. En investissant ce matériel ordinaire, nous affirmions que le féminisme fait partie intrinsèque $d u$ syndicalisme. Finalement, la multiplicité et la variété des supports d'enquête ont été payantes. Plus de 200 questionnaires ont été collectés. Des collègues se sont appropriées le support papier pour effectuer une action collective d'envois postaux groupés et probablement échanger ensemble. L'utilisation de plusieurs modalités de collecte $^{20}$ entraînait nécessairement la mobilisation de camarades diversement impliqué·es dans cette action.

35 La réutilisation et la pérennité de notre questionnaire ont été pensées dès le début du processus de création dans un continuum de partage et d'entraide. Nous l'avons réalisé sous Framaforms, un logiciel libre de création de formulaires en ligne qui permet la reproductibilité et la réutilisation des questionnaires créés. Nos modalités textuelles de traitement confidentiel, de garantie de l'anonymat et de travail en confiance, ont également été mises en ligne et rendues accessibles par toutes et tous.

Enfin, la collecte et le traitement des questionnaires papier remplis a été une occasion de travail collaboratif et de partage d'expériences. Nous nous sommes réparti les questionnaires, formées au logiciel de centralisation en ligne, au respect de la confidentialité mais aussi à l'archivage. Certains questionnaires arrivés par courrier postal étaient identifiables. Leur élimination confidentielle immédiate à l'issue de leur entrée dans l'outil centralisateur a été décidée collectivement. De même, la 
conservation de deux échantillons significatifs a été pensée et réfléchie ensemble, dans un souci de préservation à titre historique.

\section{Des affiches}

Affiche campagne LGBT 1ère vague, 2019

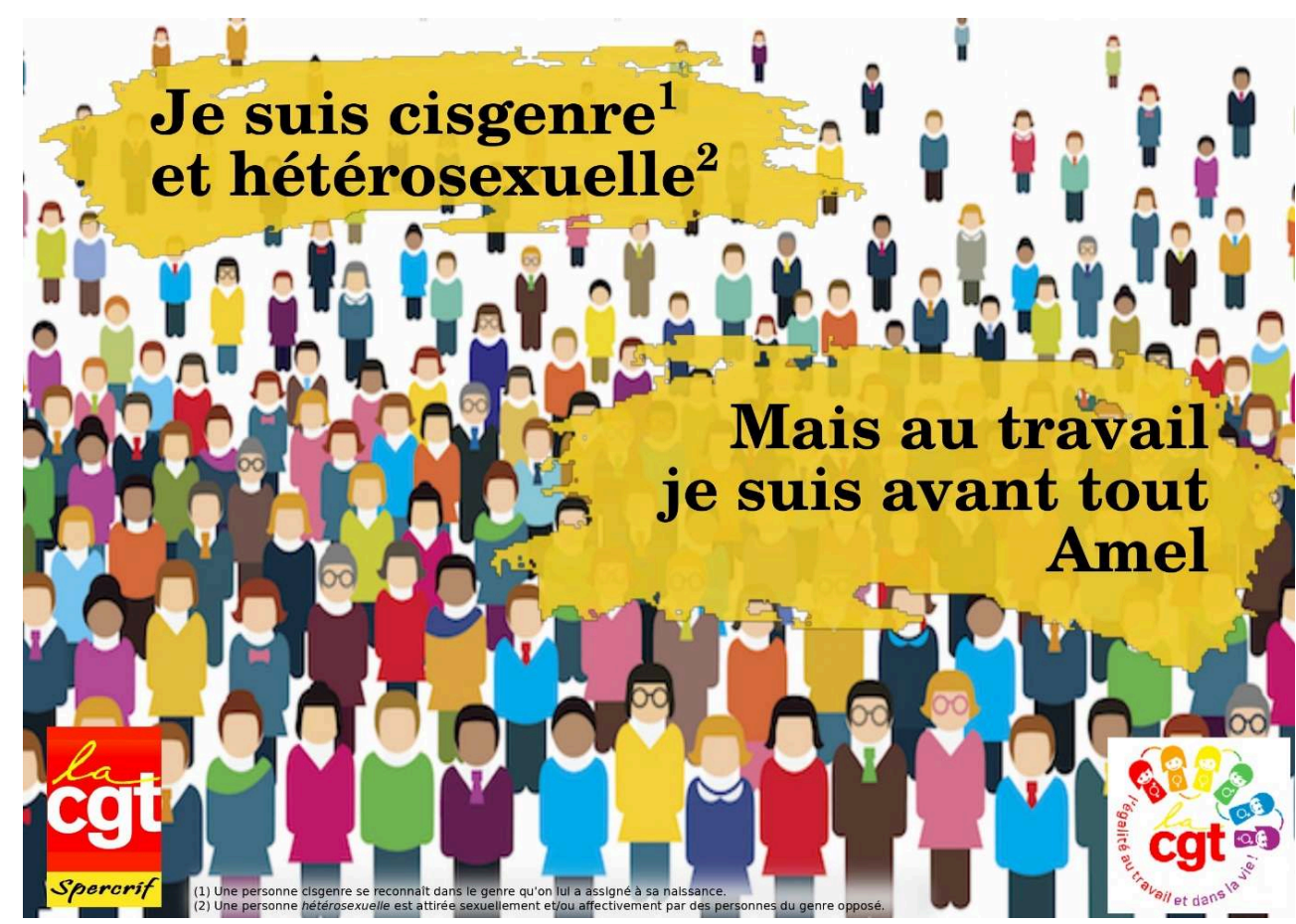

37 L'utilisation de l'affiche pour interpeler et sensibiliser les collègues aux discriminations vécues par les personnes ayant des identités sexuelles ou de genre non conformes aux normes hétéro-patriarcales (femme/homme, cisgenre, hétérosexuel-le) constitue une porte d'entrée efficace pour la construction d'un rapport de force féministe.

Outils de médiation et de visibilité, la multiplicité des affiches produites et collées (22 affiches différentes au total ; 6 pour la première vague et 16 pour la deuxième vague de notre campagne) a permis une saturation de l'espace de travail et militant. L'attention portée sur le graphisme et l'esthétique a contribué à faire sortir les affiches du paysage classique de visibilité. Nous avons pris la véritable place qui correspond à l'importance du sujet.

La campagne d'affichage a été réalisée en deux temps. Une première vague d'affiches interrogeait sur le respect au travail des identités hétéro-patriarcales. La deuxième vague, en réponse à la première, présentait les identités non conformes en accentuant l'interpellation sur leur définition politique.

Contrairement au tract, dont la durée de visibilité est réduite par l'action même de tractage, l'affiche s'inscrit dans une temporalité plus longue. Notre groupe a ainsi assumé une forme particulière de collage collectif en utilisant le débordement légitime des règles. Nous avons affiché dans les endroits les plus utilisés par les collègues et couvert l'ensemble des étages de notre bâtiment sur les pauses déjeuner. L'affiche, en tant que document d'archives ${ }^{21}$, s'installe ainsi durablement dans les mémoires. 
41 Enfin, pour sortir de l'isolement et tisser des liens durables autour du partage et de la conservation plus pérenne de nos affiches, nous avons collaboré avec le Centre Hubertine Auclert qui les a insérées dans son égalithèque en ligne ${ }^{22}$.

\section{Un wiki, entre nécessité et fragilités du numérique}

Comme l'illustrent les actions décrites et analysées précédemment, un appui systématique sur des archives externes ou auto-produites, le travail collaboratif et l'élaboration délibérative graduelle ont fait de la gestion des documents un enjeu majeur dans la pérennité de notre mode de fonctionnement. Notre approche fondée sur un recours quasi systématique à des ressources existantes mettait en lumière la nécessité de partager des résultats de collecte, analyse et sélection faites individuellement, avant même d'engager une action ou une production.

43 Au final, la quantité de documents mobilisés à un instant donné imposait de les stocker dans un espace persistant où serait mutualisé le travail de stockage et d'organisation des documents.

44 Le recours à un wiki a été retenu car il apportait une solution acceptable ou satisfaisante à de nombreux problèmes. Partant de zéro et devant absorber l'évolution de nos besoins, il était important que l'outil mis en place induise un minimum d'effets d'architecture et de structure de l'information (rubriques, formats rédactionnels, modèles documentaires, typologie, taxonomie...). En ce sens le wiki offrait la plus grande plasticité numérique tout en reposant sur un jeu extrêmement réduit de constructeurs, garant de cette plasticité. Cette rusticité, si elle trouve son pendant dans une simplicité de manipulation technique, n'induit pas d'ergonomie facilitant l'exploitation des ressources mises à disposition.

45 Nous appuyer sur le numérique tant comme technologie que comme base de supports pivots de nos productions, nous conduit nécessairement à questionner nos usages au regard de la répartition inégale des capitaux entre nous. Bien qu'aucun·e des membres du groupe ne se trouve en situation d'exclusion numérique nous restons traversées par des inégalités observables dans la plupart des collectifs militants syndicaux, matérielles et culturelles. À ce titre, le wiki ${ }^{23}$ n'a pas échappé à ces déterminations faisant du numérique à la fois un élément participant de la construction du collectif tout en risquant de le fragiliser.

46 Ce danger a été collectivement identifié dès la constitution du groupe et nous nous sommes efforcé-es d'en limiter les effets. La première mesure a été d'inclure un point dédié dans nos premières réunions, sous la forme d'un partage de connaissances et de pratiques sur un des outils que nous utilisions. Centrés sur nos propres objets, ces partages ont participé de l'appropriation des outils par lesquels nous fabriquions nos archives et du contenu et du sens que l'on décide d'y inscrire. Nous n'avons cependant pas échappé à la spécialisation de certain·es camarades pour des tâches telles que la mise à jour et la réorganisation permanente du wiki ou la production de formats terminaux de documents demandant des savoir-faire spécifiques ${ }^{24}$. Bien qu'elles se manifestent dans le numérique, ces compétences étaient mobilisées comme un bien collectif au même titre que des compétences graphiques ou artistiques dès lors qu'elles ne devenaient pas des ressorts de pouvoir mobilisés pour faire prévaloir des choix politiques. 
47 Enfin, si le wiki répond à l'enjeu de sauvegarde à court terme de nos archives militantes, la fragilité de l'outil numérique dans la pérennité de notre matrimoine interroge. La conservation des archives papier de notre groupe féministe et syndical pourrait y pallier. Elle n'a cependant pas été partagée entre les camarades, mais a reposé essentiellement sur l'une d'entre elles. Aujourd'hui nous nous interrogeons encore sur les modalités de don de nos archives, dans le même souci d'échanges et de communication de nos pratiques et de nos objets qui nous animent depuis le début de notre existence. Les réflexions communes que nous n'avons pas pu avoir, faute de temps, sur l'ouverture de nos archives, leur communicabilité et la transmission à des fins historiques et dynamiques devront être posées, quel que soit leur support.

\section{Pratiques féministes et construction du rapport de force}

48 Être féministe c'est reconnaître les rapports de classe et de domination entre les personnes. C'est dévoiler minutieusement et précisément les mécanismes de reproduction de ces rapports sociaux. C'est lutter contre l'identification d'expertes et l'utilisation de camarades comme des prestataires de services. C'est tenir compte de la matérialité des conditions d'oppression, sans exclure ou hiérarchiser. C'est donc permettre à chacune de s'exprimer, quel que soit son «bagage de départ ». Et que cette parole soit entendue et prise en compte sans distinction ou traitement différencié.

49 Être féministe c'est aussi dire «nous» au lieu de "je», sans gommer nos identités particulières, mais en s'inscrivant dans l'idée selon laquelle «faire ensemble » constitue un préalable à tout. C'est partager et s'entraider, pas nécessairement par utilitarisme individuel, mais parce que nous en avons besoin pour nous, pour exister et nous sentir fortes. C'est mettre au cœur de nos actions la sororité comme arme de déstabilisation $\mathrm{du}$ positionnement familial et patriarcal. C'est prendre du plaisir dans notre militantisme en construisant ensemble de la chaleur et de la joie, comme autant de moments de résistance face à nos souffrances quotidiennes.

Être féministe c'est gagner collectivement en confiance et en apprentissage. C'est rester humble dans la subversion et dans l'occupation individuelle de l'espace. C'est enfin remettre en cause, interroger nos pratiques et les éprouver de façon permanente.

51 À partir de la théorie, nous avons tenté de construire un rapport de force féministe en interne de notre organisation et vis-à-vis de notre employeur en nous appuyant notamment sur l'élaboration, l'utilisation et la conservation de nos objets d'archives militants.

52 La quantité des outils militants décrits éclaire un aspect des enjeux politiques et stratégiques que nous poursuivions. L'occupation régulière de l'espace inscrit notre action et notre présence dans le temps long, construisant ainsi une forme de permanence tant pour ses effets agitatoires que légitimants. Il s'agissait de rendre familière pour les collègues une parole publique, militante, syndicale posant des mots sur les violences et les inégalités à l'encontre des femmes ${ }^{25}$, les dénonçant sans relâche et portant des revendications capables de les réduire. Si ce principe est en lui-même applicable à tout terrain revendicatif, le mettre en œuvre dans l'action féministe et syndicale n'est rien d'autre que donner, dans les faits, au féminisme la place qu'il devrait occuper dans toutes les luttes sociales, ce qui est loin d'être le cas aujourd'hui. 
Sans revenir sur les raisons de ce déficit, il est le produit et il participe de la délégitimation des luttes féministes ${ }^{26}$ tant dans nos organisations syndicales qu'auprès des collègues.

53 En termes qualitatifs, nous nous sommes attaché à ce que nos productions et nos actions aient une forte visibilité dans l'espace où nous les avons déployées. La diversité et l'originalité de nos objets d'archives militants répondaient à cet enjeu. Nous voulions également faire corps dans nos pratiques, ou faire chorus comme dans l'interprétation de notre chanson.

Quelle que soit l'appréciation que l'on puisse porter sur la qualité de notre travail militant, nous sommes finalement convaincues que sa compréhension et son élaboration doivent autant à l'héritage féministe dont nous nous sommes nourries que de notre propre contribution.

\section{BIBLIOGRAPHIE}

CONNELL, Raewyn. 2014 [1995]. Masculinités. Enjeux sociaux de l'hégémonie. Paris : Amsterdam Éditions

FOUCAULT, Michel. 1969. L'archéologie du savoir. Paris : Gallimard.

GUILLAUMIN, Colette. 2016 [1992]. Sexe, race et pratique du pouvoir. L'idée de nature. DonnemarieDontilly : Éditions iXe.

\section{NOTES}

1. Nous inscrivons notre propos dans la définition des archives donnée par le Code du patrimoine: «Les archives sont l'ensemble des documents, y compris les données, quels que soient leur date, leur lieu de conservation, leur forme et leur support, produits ou reçus par toute personne physique ou morale et par tout service ou organisme public ou privé dans l'exercice de leur activité. » (article L211-1). Nous entendons dans cette définition, qu'un document est une archive dès sa création. Ce statut englobant ne signifie pas pour autant que toutes les archives portent une "valeur archivistique », entendue comme informative, juridique ou historique. Nous considérons que l'ensemble de nos documents portent a minima l'une de ces trois valeurs, en eux-mêmes ou en corrélation explicite avec d'autres.

2. CONNELL, Raewyn. 2014 [1995]. Masculinités. Enjeux sociaux de l'hégémonie. Paris : Amsterdam Éditions.

3. Les réunions des commissions exécutives de notre syndicat peuvent régulièrement être coupées de la mémoire d'une action. Certains sujets sont présentés comme sans histoire, inscrits à l'ordre du jour de manière arbitraire.

4. Chaque sujet est à un moment donné de l'action syndicale traversé par plusieurs approches dont certaines acquièrent un caractère dominant. La singularité des analyses et angles privilégiés par tout collectif militant ne peut être maintenue et enrichie qu'en se préservant de cette domination qui agit à chaque instant. 
5. Lorsqu'on arrive dans notre syndicat, il n'y a pas de présentation systématique de son mode de fonctionnement, des outils d'organisation existants. Ce savoir et cette connaissance dépendent des camarades. La découverte des pratiques syndicales se réalise de façon individuelle et au gré des relations que la ou le camarade entretient avec les membres « chevronnées ».

6. FOUCAULT, Michel. 1969. L'archéologie du savoir. Paris : Gallimard.

7. De même que dans les sphères domestiques et professionnelles le travail des femmes est approprié par les hommes, comme l'ont analysé, dès les années 70, plusieurs théoriciennes féministes telle la sociologue Collette Guillaumin (GUILLAUMIN, Colette. 1978. «Pratique du pouvoir et idée de Nature (1) L'appropriation des femmes" Questions féministes (2), Les corps appropriés : 5-30).

8. Par exemple: la non-démocratie revendiquée (la famille n'est pas une organisation démocratique), l'harmonie factice par silenciation des oppositions et oppressions, le secret des divergences, y compris vis-à-vis des syndiqué·es, la figure tutélaire du secrétaire général...

9. Une campagne vise notamment à maîtriser les axes de revendications définis collectivement et déployés sur un temps relativement long. Maintenir le cap et ne pas laisser dépérir ce travail d'élaboration est un enjeu.

10. Éviter la segmentation entre conception d'une action, production des outils militants et mise en œuvre de ces outils sur le terrain.

11. Le choix du féminin exprime le fait qu'à ce moment là de l'existence de notre groupe, seules les femmes étaient actives.

12. TISSOT, Florence. TISSOT, Sylvie. 2015. Je ne suis pas féministe mais... France. Les mots sont importants.

13. Il s'agit à la fois d'un article d'une militante féministe, de vidéos féministes (Arte vidéo), d'extrait de podcast féministe (Les couilles sur la table, épisode sur le genre du capital), etc.

14. Le fait que le recours à un dispositif technique n'induise pas de discrimination plus importante que celle que nous souhaitions éviter fut à la fois le fruit de l'apprentissage collectif en matière de capture et d'échange de sources sonores et de la confiance en nous acquise à travers cette expérience.

15. https://video.antopie.org/videos/watch/fa0d2d4a-1992-48b5-9b25-f9252e9877f2 (consulté le 10 mai 2021)

16. http://www.egalite-professionnelle.cgt.fr/les-10-enseignements-a-retenir/ (consulté le 10 mai 2021)

17. Collectif confédéral femmes et mixité de la CGT.

18. CGT Educ'Action 93.

19. Dans les lycées, travaillent des agent·es de la fonction publique territoriale et de l'éducation nationale.

20. De la main à la main, par mail à des militant·es ou des structures syndicales, par courrier postal, par dépôt dans les locaux syndicaux.

21. Notre affiche devient archive dès sa création.

22. https://m.centre-hubertine-auclert.fr/outil/affiche-sensibilisation-lgbt-cgt (consulté le 10 mai 2021)

23. Comme d'autres outils participant de notre fonctionnement quotidien, tel l'outil de rédaction collaborative simultanée (etherpad).

24. Impressions en haute qualité, montage et reformatage de contenus vidéos ou sonores...

25. Également, dans une moindre mesure, des personnes lgbti.

26. Qui se limitent souvent à des listes revendicatives incantatoires. 


\section{RÉSUMÉS}

Groupe féministe au sein d'un syndicat, la production d'archives, entendues comme traces de notre activité militante revêt une triple dimension politique.

En tant qu'outils matériels de description et de représentation d'une action politique et féministe, les archives inscrivent dans le temps, la réalité et la matérialité de nos engagements et de nos réalisations. Dans un contexte social et syndical marqué par des rapports de domination masculine qui n'échappent pas au fonctionnement et à l'activité militante, les archives constituent des sources fondamentales de légitimation de nos actions féministes. Enfin, le processus de production des archives de notre groupe constitue en soi une pratique féministe.

À travers des exemples d'objets militants, d'outils et de supports numériques, nous illustrons, dans une démarche réflexive, la construction d'un rapport de force féministe singulier. Les conditions de création, les usages et les modalités de conservation de nos archives témoignent de pratiques politiques réfléchies, toutes animées en faveur de la lutte contre le patriarcat.

A feminist group within a union, and the production of records, understood as traces of our militant activity, has a triple political dimension.

As material tools for the description and representation of political and feminist action, records inscribe in time, the reality and the materiality of our commitments and our achievements. Despite the fact that the context is a social and union one, it is nonetheless marked by relationships of male domination in its functioning and militant activity. Records and archives therefore constitute fundamental sources of legitimization of our feminist actions. Finally, the process of production of our group's records constitutes in itself a feminist practice.

Through examples of activist objects, digital tools and formats we illustrate the construction of a singular feminist power struggle. The conditions of creation, and the uses and the methods of preservation of our archives demonstrate deliberate political practices, all with the objective of fighting against the patriarchy.

\section{INDEX}

Thèmes : Explorations

Keywords : feminism, unionism, records, power struggle, digital tools and formats

Mots-clés : féminisme, syndicalisme, archives, rapport de force, outils et supports numériques

\section{AUTEURS}

MAËVA BALLON

Militante féministe et syndicale

\section{FLAZ TORRES}

Militante féministe et syndicale 